\title{
Is Male Infertility a Forerunner to Cancer?
}

\author{
Whitney R. Burns, Edmund Sabanegh, Rima Dada, Brandon Rein, Ashok Agarwal \\ Center for Reproductive Medicine (WRB, ES, RD, BR, AA), Glickman Urological and Kidney Institute, \\ Cleveland Clinic, Cleveland, Ohio, USA and All India Institute of Medical Sciences (RD), New Delhi, \\ India
}

\begin{abstract}
Purpose: The frequency of testicular cancer and male infertility has been increasing in the past several decades. This article examines the relationship between male infertility and testicular cancer, concentrating particularly on causal links.

Results: Both of these disorders are associated with testicular dysgenesis syndrome and have also been traced to mutations in genes involving DNA repair and tumor suppression, as well as environmental exposure.

Conclusion: The identification and examination of these common points of origin supports the integration of testicular cancer screenings into the routine evaluation of infertile men.
\end{abstract}

Key words: male infertility; endocrine-disruption; testicular cancer; testicular dysgenesis

Int Braz J Urol. 2010; 36: 527-36

\section{INTRODUCTION}

In recent years there has been a marked decline in male reproductive health. Concomitant with a decrease in semen quality, there has been a 3- to 4-fold increase in the incidence of genitourinary abnormalities and a 6-fold increase in the incidence of testicular cancer. Declines in semen quality have caused an increase in the population of infertile males. Recent studies have also shown that there is an increase in the incidence of gonadal tumors in infertile men. Therefore, there has been a growing concern that infertility may actually be a forerunner to or an early marker of testicular cancer.

Infertility and testicular cancer have been associated with one another as well as with genitourinary abnormalities including, cryptorchidism and hypospadias, and this triad of disorders constitutes a testicular dysgenesis syndrome (TDS). In this review, we will explore the various causal links between the components of TDS. The purpose of this article is to fortify the connection between infertility and testicular germ cell tumors by identifying their common origin, describing the incidence of disease states linking the two, and connecting carcinoma-in-situ (CIS) testis to both infertility and testicular cancer.

\section{ETIOLOGY OF TESTICULAR CANCER}

\section{Risk Factors}

Although many potential risk factors for germ cell tumors have been proposed, few have attained high levels of evidence in support of their association with testicular malignancies. Thus far, only cryptorchidism, contralateral testicular germ cell tumor, and familial history of testicular cancer have proven to be sound predictors of testicular cancer risk (1). A meta-analysis of 20 case control studies conducted by 
Dieckmann and Pichlmeier found the relative risk of testicular germ cell tumors in individuals with a history of undescended testes to be 5 times greater than that of men without that history (1). In individuals with cryptorchidism, the risk of testicular cancer is also increased for the contralateral testes, providing support for the importance of testicular dysgenesis as a common point of origin (1). Familial history of testicular cancer confers a relative risk of 3-10, perhaps as a consequence of the inheritance of a susceptibility gene that has been identified on the Xchromosome (1). Additionally, patients with unilateral testicular germ cell tumors possess a much greater risk of developing a contralateral germ cell tumor (1). Three other factors twinship, testicular atrophy, and infertility, have also been associated with testicular germ cell tumors, but, so far, the evidence for these relationships has not been substantiated (1).

\section{Carcinoma-in-situ Testis}

Carcinoma-in-situ of the testis precedes the formation of all but two very rare forms of testicular cancer (2). In fact, fifty-percent or more of those individuals diagnosed with CIS will develop invasive testicular cancer within five years (2).

Genetically, CIS cells resemble embryonic stem cells, with their gene expression profile closely mirroring that of primordial germ cells and gonocytes but not that of infantile spermatogonia or adult germ cells (3). The proteins expressed in CIS cells are normally down-regulated during the transition from normal gonocytes to spermatogonia, suggesting that the origin of CIS cells is pre-meiotic in nature. It has been hypothesized that this is not the result of an inherent germ cell abnormality but, rather, inappropriate age signaling from surrounding cells, particularly Sertoli and Leydig cells, allowing continued germ cell expression of pluripotency and self-renewal genes. Gene up-regulation could also be the result of a failure of abnormal germ cells to undergo apoptosis. Both escape of cell cycle check points and improper age signaling could allow clonal proliferation of these abnormal cells, resulting in cells similar to those described in CIS.

Because fewer gonocytes are fully maturing to become spermatozoa in these instances, the total sperm count of men with CIS is lower, possibly resulting in sub-fertility. In support of this claim, impaired spermatogenesis has been observed in testes diagnosed with CIS (2). Similarly, the incidence of CIS in infertile men is $4-1.1 \%$ (4). This is strongly supportive of a link between infertility and testicular cancer.

\section{MALE INFERTILITY}

Male infertility and impaired spermatogenesis serve as one of the pillars of TDS. Infertility affects roughly $15 \%$ of couples, with $50 \%$ of the cases resulting from male factor (5). While the incidence of male infertility continues to rise, the majority of causes remain idiopathic. Both non-genetic and genetic factors contribute by influencing physiological processes related to spermatogenesis.

\section{Genetic Causes}

The most severe presentations of TDS, those that result in hypospadias, cryptorchidism, subfertility, and testicular cancer, are associated with genetic causes (6). In addition, genetic abnormalities are associated with approximately $15-30 \%$ of male infertility cases (7). Both of these disorders have been described in individuals with androgen insensitivity, 45X/46XY karyotype, and SRY mutations (6). Male infertility and testicular cancer have also been associated with defects in DNA repair genes, tumor suppressor gene mutations, and epimutations (8).

The 45X/46XY karyotype has been associated with a variety of medical conditions. Ambiguous genitalia and mixed gonadal dysgenesis are key features of this disorder (9). These patients are at increased risk for gonadal tumors, impaired fertility, and fibrosis as a result of ongoing gonadal changes (10). Most instances of this type of mosaicism are the result of a structural abnormality of or loss of the $\mathrm{Y}$ chromosome, specifically through nondisjunction following normal fertilization.

Mutations in the SRY gene have also been linked to gonadal tumor formation and infertility. Alterations of SRY are most commonly associated 
with complete gonadal dysgenesis. These patients can present with phenotypes ranging from streak gonads like those seen in Turner syndrome to genital ambiguity. One meta-analysis noted gonadal tumor formation in $52.5 \%$ of patients with SRY abnormalities (11).

Spermatogenic impairment, infertility, and testicular cancer formation can also be associated with defects in DNA repair genes. Supraphysiological reactive oxygen species (ROS) levels in the semen have been well established to lead to oxidative damage to the sperm, which manifests as DNA breakage, cross-linkage, and mutations (12). Recent studies have shown that infertile men possess high ROS levels (12). In addition to the DNA damage caused, ROS also results in the production of highly mutagenic compounds that can further increase an individual's susceptibility to tumor formation (12).

Persistence of this damage is indicative of a deficiency in the DNA repair mechanisms. In addition, mutations in these repair genes can result in deletions or expansions of small repeat DNA sequences, unstable components that have been noted in many forms of cancer (13). Furthermore, these mutations and expansions of DNA repeat sequences can manifest as male infertility (13).

Cases of male infertility and cancer formation have also been attributed to deficiencies in tumor suppressor genes, particularly p53. Known to have a crucial role in tumor prevention and stress response pathways, p53 normally aides in coordinating a variety of cellular responses from cell cycle arrest and apoptosis to the maintenance of genomic stability. This gene also has an important role in spermatogenesis, specifically aiding in prophase of meiosis within primary spermatocytes. Mutations result in chromosomal and genomic instability, increasing the chance that p53 null cells will become malignant in nature and gain additional mutations (14).

Additionally, it has also been reported that p53 has a role in the up-regulation of certain antioxidant genes (15). Therefore, a mutation of this gene would certainly lead to higher levels of ROS, comparable to those seen in infertile males. Although there have been very few studies in humans on the effects of p53 mutations, knockout mice are infertile.

\section{TESTICULAR DYSGENESIS SYNDROME}

Testicular dysgenesis syndrome is one of several possible male reproductive health complications that could result from an aberrant developmental pathway. The components of TDS include low sperm count, hypospadias, cryptorchidism, and testicular cancer.

\section{Incidence}

The presentation of TDS is variable, with each of the symptoms present in differing degrees or one or more of them being absent entirely. Mild TDS has been estimated to affect $20 \%$ of the world's male population, while $5 \%$ of the population is thought to be afflicted with a more severe presentation in which all of the symptoms are present to a significant degree (16).

The increasing frequency of the individual health problems of TDS may suggest that these numbers are only going to get larger in the coming years. A recent study of eight populations with a long history of cancer registration saw the incidence of testicular cancer double in a twenty year period (17). In the same timeframe, there has been a marked decline in sperm density (18). Cryptorchidism and hypospadias are following a comparable temporal incline (19). These similarities have been taken as evidence for their linkage.

\section{Evidence for the Linkage of the Three Presentations of TDS}

In addition to these temporal trends, there have been many studies, especially from Scandinavian countries, that report geographic concentrations of these reproductive health problems. In Denmark, for example, twenty percent of the male population has suboptimal semen parameters, while sperm counts in nearby Finland have remained at a high level for the past few decades (20). In support of the existence of TDS, Danish men also exhibit higher incidences of congenital cryptorchidism and hypospadias, as well as a greater frequency of testicular cancer, than do Finnish men (21-23). 


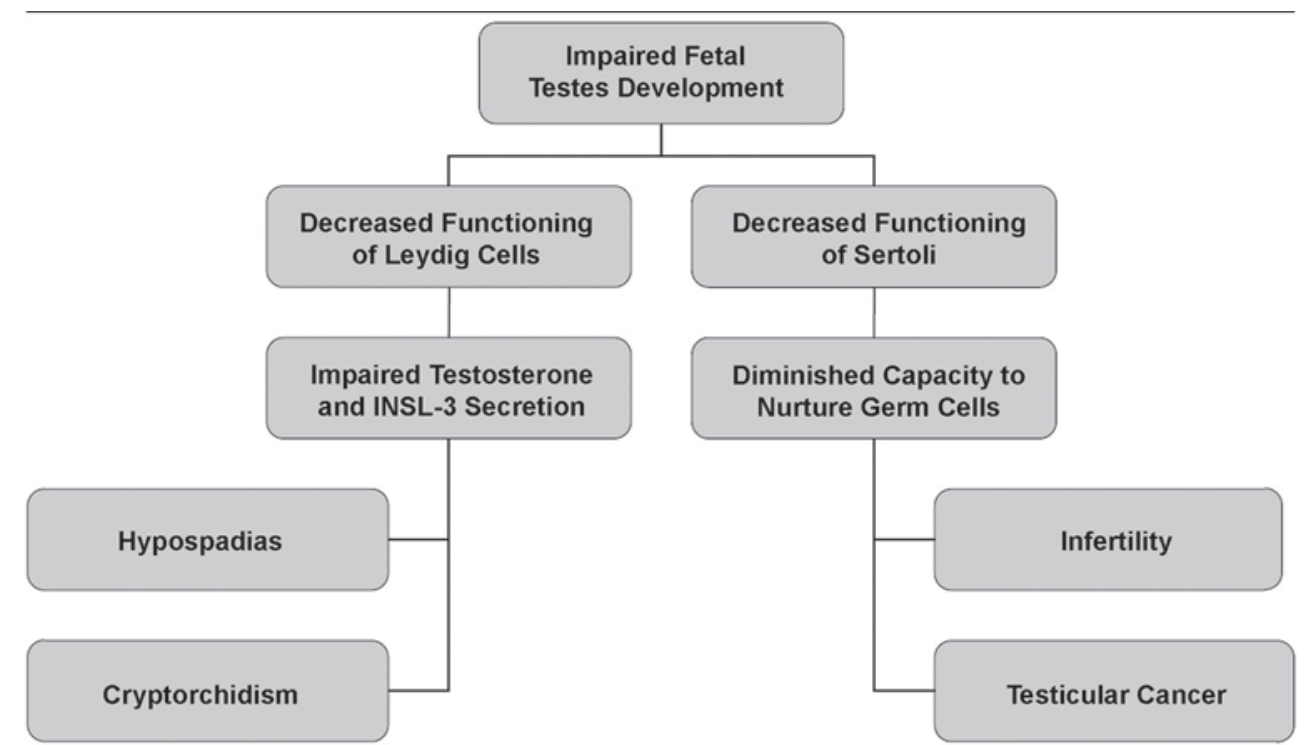

Figure 1 - Proposed mechanism leading to testicular dysgenesis. In this pathway, the key event is the impairment of testes development during fetal life.

Other studies have found very strong associations between two or more of the reproductive health disorders associated with TDS. For example, men with a history of cryptorchidism have a risk of testicular cancer that is 2-8 times that of those men without a history of undescended testes (1). Additionally, cryptorchidism is associated with impaired spermatogenesis and spermatogenic arrest, resulting in infertility (24). The congenital birth defects, cryptorchidism and hypospadias, which are manifestations of TDS are also very closely related to one another (25). The connection between infertility and testicular cancer is one that is still disputed, but many recent studies have found evidence to suggest that this link is particularly strong.

As suggested by the name of the disorder, testicular dysgenesis is the underlying correlation between the four presentations of this disease. Evidence of testicular dysgenesis in biopsies from patients with infertility or testicular germ cell tumors includes the presence of immature seminiferous tubules with undifferentiated Sertoli cells and Sertoli cell-only tubules (6). There have been several mechanisms proposed to explain the origins of these features, all of which rely upon the disruption of normal male differentiation during fetal development.

\section{Proposed Pathway Leading to Testicular Dysgenesis}

As the result of some genetic or environmental influence, normal development of the testes is impaired (Figure-1), resulting in improper functioning of the Sertoli and Leydig cells (26). The potential causes of this interruption are discussed below .

Improper Leydig cell function results in deficiencies in both testosterone and INSL-3 secretion. These two hormones are responsible for testicular descent. Therefore, it is highly likely that a disruption in their production could lead to cryptorchidism. In addition, because testosterone is the key hormone involved in the formation of male external genitalia, low testosterone levels lead to improper fusion of the urethral folds and hypospadias.

Intact and competent Sertoli cells are required for optimal spermatogenesis and spermiogenesis. Therefore, improper functioning of these cells leads to hypospermatogenesis and infertility (6). Additionally, the malfunctioning of Sertoli cells leads to the arrest of many gonocytes at an early stage of maturation. These arrested gonocytes are thought to be the forerunners to carcinoma in situ testis which, in turn, can develop into testicular germ cell cancer. Additionally, as a 
consequence of decreased testosterone level, there will also be a diminished number of Sertoli cells, thereby compounding the problems associated with their malfunction.

The mechanism whereby testes development is interrupted remains to be identified, but both genetic and environmental factors have been implicated. The genetic causes have already been discussed in great length above. Therefore, an examination of environmental factors will be the main focus below.

\section{Environment Factors and Testicular Dysgenesis}

As previously mentioned, all the male reproductive health problems associated with TDS have greatly increased in frequency in the past several decades. A higher risk of testicular cancer and poor semen quality are closely associated with one's year of birth $(27,28)$. This cohort phenomenon is strongly suggestive of some degree of environmental influence at work.

Environmental variables could be on the macroscopic scale. For example, geographic location of a mother during pregnancy can be indicative of her son's risk of TDS (6). Insults to the micro-environment of the fetus, however, can also play a substantive role in the development of the disease.

The environmental variable that is currently receiving the most attention as a possible mediating factor in the development of testicular dysgenesis is a group of compounds known as endocrine-disrupting chemicals or xenoestrogens. These are anti-androgenic agents which mimic estrogens.

\section{Endocrine Disrupting Chemicals}

Phthalates, gums, and paints are anti-androgenic chemicals that are ubiquitous in human life. They have been used as plasticizers in PVC products and are constituents in many infant toys, storage containers, and medical devices (29). While experts recommend that daily exposure be limited to $2 \mathrm{mg}$, with nearly 18 billion pounds of phthalates being produced per year, many individuals have occupational or medical exposures greatly in excess of these guidelines (29).

Although humans are generally exposed to the diester form of phthalates (Figure-2), their more pressing concern should be the monoester forms that these chemicals are converted into within the body. This monoester form is 10 times more toxic than the diester form, and has been found in detectable levels in $75 \%$ of the population, thereby greatly increasing the risk for male reproductive tract disorders like those of TDS (29).

\section{Proposed Mechanism}

These agents are believed to cause an increase in estrogen levels in the blood which, in turn, inhibits the hypothalamopitutary gonadal axis, resulting in decreased production of follicle stimulating hormone (FSH) and, subsequently, a fixing of Sertoli cell number.

In most mammals, Sertoli cell replication occurs only during fetal and post natal life. Sertoli cell number thus becomes fixed at a particular stage of development. However, in man, the Sertoli cell number increases significantly between late fetal and pre-pubertal life and also increases further during puberty. Hence the window for adverse effect on Sertoli cells in man is longer than that known for other mam-

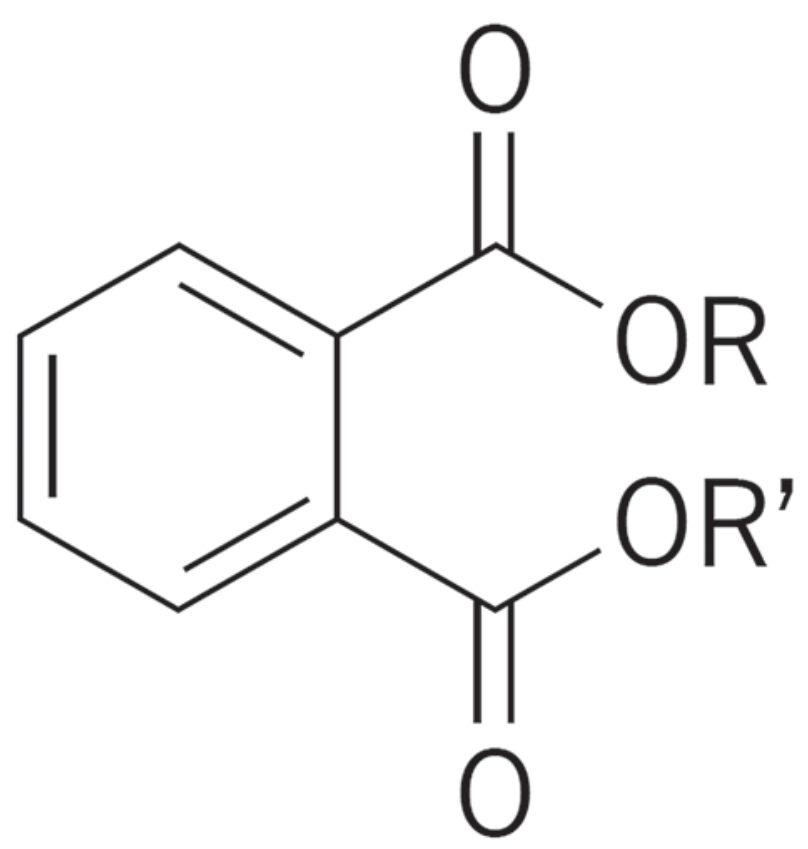

Figure 2-Generic diester phthalate. 
Is Male Infertility a Forerunner to Cancer?

Table 1 - Hormonal changes indicative of Leydig and Sertoli cell malfunction in testicular dysgenesis syndrome (TDS).

\begin{tabular}{lccc}
\hline & FSH & LH & Serum Testosterone \\
\hline Cryptorchidism & Elevated & Elevated & $--^{*}$ \\
Hypospadias & Elevated & Elevated & $-^{*}$ \\
Infertility & $--^{*}$ & Elevated & Decreased \\
Testicular Cancer & Elevated & Elevated & Decreased \\
\hline
\end{tabular}

Changes in hormonal Assays with the reproductive health problems of TDS; * Indicates where data was unavailable; FSH = follicle stimulating hormone; $\mathrm{LH}=$ luteinizing hormone.

malian species. Thus, after exposure to environmental hormones, xenoestrogens, or environmental endocrine disruptors, these chemicals accumulate in the body, and their effects are biomagnified over a period of time.

Upon exposure to an endocrine disruptor, the resulting elevation in maternal and fetal estrogens inhibits FSH secretion, leading to decreased Sertoli cell number, lower levels of anti-Mullerian hormone, and decreased expression of SRY. This may result in abnormal sexual differentiation, leading to cryptorchidism and hypospadias, as well as hypospermatogenesis $(30,31)$. It also eventually may lead to testicular cancer through a process known as hormonal carcinogenesis (32-34).

These findings were summarized by, Sharpe and Skakkebaek in a landmark article, now known as the "estrogen hypothesis", which linked exposure to exogenous estrogens to male reproductive tract disorders, including TDS (35).

While their precise effect on the male reproductive tract is poorly understood, phthalates and other endocrine-disrupting chemicals have been strongly correlated with each of the reproductive health problems associated with TDS in both human and animal studies. As this research becomes more thorough, our understanding of the mechanisms from which these disorders result will also become more clear.

\section{Evidence from Human Retrospective Studies}

Human retrospective studies have provided considerable evidence for the importance of endocrine disrupting chemicals in the induction of male reproductive health disorders. One potent source of synthetic estrogen exposure is pesticides, which are known to possess estrogenic, anti-androgenic, and aromatase inhibiting effects in vivo. It has been shown that maternal exposure to pesticides during pregnancy is associated with an increased risk of cryptorchidism in male infants (36). A separate study also found that women who worked in greenhouses while pregnant gave birth to sons with significant reproductive health impairment, particularly a three-fold greater risk of cryptorchidism (37).

Studies investigating the different hormone levels in males exhibiting one or more reproductive health problems have also been helpful in demonstrating the possible pathogenesis of TDS. Three month old male infants with cryptorchidism were shown to have significantly elevated levels of FSH and LH as well as reduced inhibin B concentrations compared with controls (38). Similar changes in hormone levels were also seen in boys with hypospadias (39). Additionally, lower serum testosterone levels have been reported in infertile men compared with proven fertile men. Infertile men in this study had a lower testosterone/LH ratio and higher serum LH levels as well (40). Higher levels of LH and FSH, in addition to lower serum testosterone levels, have also been seen in men with CIS and testicular cancer (41), Table-1.

Taken together, these hormonal changes are indicative of compensated Leydig cell failure. The pituitary gland releases LH to stimulate the Leydig cells to produce testosterone. When Leydig cells fail to properly receive or interpret this signal, the pituitary secretes more LH to compensate, resulting in high levels of LH and low levels of testosterone in the blood. Decreased androgen production that would accompany Leydig cell malfunctioning has recently 
been shown to have a dramatic effect on the number of Sertoli cells in the perinatal period. Faulty nurse cells will be less capable of nurturing germ cells into mature spermatozoa, leading to poor semen quality in adulthood and also to germ cell maturation arrest, increasing the likelihood of developing CIS and testicular cancer. These changes are exactly those hypothesized to be involved in the pathogenesis of TDS $(29,42,43)$.

\section{Evidence from Animal Models}

Several animal models have concentrated on the effects of different endocrine-disrupting compounds on male reproductive tract development in rats or mice. In a study published by Fisher et al., exposure to dibutyl phthalate in utero resulted in the abnormal function and distribution of all major cell types within the rat testis (44). This study described genital abnormalities at both the macroscopic and microscopic level that are similar to those disorders associated with TDS in humans, including cryptorchidism, hypospadias, incomplete seminiferous cord formation, and Sertoli cell-only tubules. Additionally, changes in the proportion of Sertoli cells seen within those males exposed to DBP rendered germ cells incapable of maturation and support of spermatogenesis (44). A similar study by Mahood et al., in which male rats were exposed to DBP in utero, resulted in decreased INSL-3 gene expression, multi-nucleated gonocytes, abnormal Leydig cell aggregation, and decreased testicular testosterone (45). The results of these two studies are indicative of severe testicular dysgenesis as a result of in utero exposure to endocrine-disrupting chemicals. Additional studies on rodents have shown similar results $(29,42,43)$.

Because the negative effects of exposure to these chemicals are not evenly distributed among the treatment groups, there likely exists some degree of genetic predisposition for developing the male reproductive disorders associated with exposure. These findings lend credence to the assumption that TDS is a multi-factorial disease involving both a genetic and environmental contribution.

While animal models have provided much mechanistic insight into the role of endocrine disrup- tors in TDS, they have yet to observe testicular cancer in the treated animals. Therefore, the current belief that testicular cancer is connected to both the genital abnormalities and infertility attributed to TDS is reliant upon human retrospective studies and our current understanding of the fetal origins of these disorders and of testicular cancer itself.

\section{TESTICULAR CANCER AND MALE INFERTILITY}

Testicular dysgenesis syndrome proposes an association between testicular cancer, male infertility, and genital abnormalities. Due to the long time period over which these various disorders may appear, it is difficult to track the course of those potentially afflicted individuals longitudinally. More easily done, however, is to study the associations between testicular cancer and infertility, two male reproductive disorders that present at roughly the same time in an individual's life. The establishment of a definitive relationship between these two diseases could significantly decrease the incidence of testicular cancer if this association was indicative of a benefit to increased testicular cancer screening for infertile patients. Thus far, there have been many studies within the literature that focus on this relationship specifically.

Some studies have looked retrospectively at the pre-cancer fertility capacity of men that later developed testicular cancer. For example, it has been shown that men develop testicular cancer have fewer children than age-matched men who did not develop testicular cancer (46). Another study by Petersen and Skakkebaek analyzed the semen quality of men diagnosed with unilateral testicular cancer. This group reported that the quality of semen collected from these men was much poorer than would typically be expected for a man possessing one functional testicle (47). CIS is also seen more frequently in the testicular biopsies of men evaluated for sub-fertility or infertility (48). This would strongly suggest that men with fertility problems, even those that go undiagnosed with sub-fertility, are at an increased risk of developing testicular cancer.

Recent research has supported the existence of this increased risk, although the exact impact of 
infertility or sub-fertility on testicular cancer risk is still disputed. A study by Jacobson et al. reported the increased risk of testicular cancer in men with fertility problems to be 1.6 times that of men with normal semen parameters (49). A more recent study by Raman estimated the incidence of testicular cancer in infertile men to be 20 times greater than that of proven fertile men (50).

\section{Clinical Implications}

The increased risk of testicular cancer associated with male infertility speaks strongly for the importance of incorporating testicular cancer screening into the routine care of infertile men. While the only definitive way to determine if testicular cancer is present is to perform a testicular biopsy, this test is invasive and may not be a practical way to screen a large population of infertile males. Scrotal ultrasonography can also detect the presence of tissue irregularities that might be indicative of testicular cancer or CIS. Perhaps the easiest way to provide testicular cancer screening to those suffering from male infertility is to teach them how to perform self-examinations. This would allow the patient to detect palpable changes in the testicular tissue that might precede testicular cancer.

\section{CONCLUSION}

The identification of testicular dysgenesis syndrome has established a connection between male infertility and testicular cancer. The reproductive health problems associated with this disorder are manifestations of improper testicular development during fetal life. Subsequent malfunctioning of both the Sertoli and the Leydig cells are responsible for the failure of gonocytes to mature properly, resulting in a lower number of mature spermatozoa and a greater likelihood of developing CIS. This common etiology, when combined with what is known about the genetic origins of male infertility and testicular cancer, is strongly suggestive of the link between these two disease states. As further research explores the relative influence of both genetics and the environment in the etiology of TDS and strengthens the connection between infertility and testicular cancer, the current research base provides compelling evidence for increasing testicular cancer screenings for infertile men. By integrating screenings into the routine evaluation of infertile men, it will be possible to detect cases of testicular cancer earlier, and, ultimately, decrease its incidence.

\section{ACKNOWLEDGEMENT}

Research supported by the Center for Reproductive Medicine, Cleveland Clinic.

\section{CONFLICT OF INTEREST}

None declared.

\section{REFERENCES}

1. Dieckmann KP, Pichlmeier U: Clinical epidemiology of testicular germ cell tumors. World J Urol. 2004; 22: 2-14.

2. Rørth M, Rajpert-De Meyts E, Andersson L, Dieckmann KP, Fossa SD, Grigor KM, et al.: Carcinoma in situ in the testis. Scand J Urol Nephrol Suppl. 2000; 205: 166-86.

3. Almstrup K, Hoei-Hansen CE, Wirkner U, Blake J, Schwager C, Ansorge W, et al.: Embryonic stem celllike features of testicular carcinoma in situ revealed by genome-wide gene expression profiling. Cancer Res. 2004; 64: 4736-43.

4. Cortes D, Thorup J, Frisch M, Møller H, Jacobsen GK, Beck BL: Examination for intratubular germ cell neoplasia at operation for undescended testis in boys. J Urol. 1994; 151: 722-5.

5. Poongothai J, Gopenath TS, Manonayaki S: Genetics of human male infertility. Singapore Med.J. 2009; 50: 336-347.

6. Wohlfahrt-Veje C, Main KM, Skakkebaek NE: Testicular dysgenesis syndrome: foetal origin of adult reproductive problems. Clin Endocrinol (Oxf). 2009; 71: 459-65.

7. Ferlin A, Raicu F, Gatta V, Zuccarello D, Palka G, Foresta C: Male infertility: role of genetic background. Reprod Biomed Online. 2007; 14: 734-45. 
8. Paul C, Povey JE, Lawrence NJ, Selfridge J, Melton DW, Saunders PT: Deletion of genes implicated in protecting the integrity of male germ cells has differential effects on the incidence of DNA breaks and germ cell loss. PLoS One. 2007; 2: e989.

9. Achermann JC, Jameson JL: Disordes of Sex Development. In: Fauci AS, Kasper DL, Hauser SL, Longo DL, Jameson JL, Loscalzo J (eds.), Harrison's Principles of Internal Medicine. New York, Mc Graw Hill. 2008; $17^{\text {th }}$ ed., pp. 2339.

10. Baskin LS: Abnormalities of Sexual Determination \& Differentiation. In: Tanagho EA, McAninch JW (eds.), Smith's General Urology. New York, Mc Graw Hill. 2008; $17^{\text {th }}$ ed., pp. 649.

11. Uehara S, Hashiyada M, Sato K, Nata M, Funato T, Okamura K: Complete XY gonadal dysgenesis and aspects of the SRYgenotype and gonadal tumor formation. J Hum Genet. 2002; 47: 279-84.

12. Hughes CM, Lewis SE, McKelvey-Martin VJ, Thompson W: A comparison of baseline and induced DNA damage in human spermatozoa from fertile and infertile men, using a modified comet assay. Mol Hum Reprod. 1996; 2: 613-9.

13. Devouassoux-Shisheboran M, Mauduit C, Bouvier R, Berger F, Bouras M, Droz JP, et al.: Expression of hMLH1 and hMSH2 and assessment of microsatellite instability in testicular and mediastinal germ cell tumours. Mol Hum Reprod. 2001; 7: 1099-105.

14. Fenton RG, Longo DL: Cancer Cell Biology and Angiogenesis. In: Fauci AS, Kasper DL, Hauser SL, Longo DL, Jameson JL, Loscalzo J (eds.), Harrison's Principles of Internal Medicine. New York, Mc Graw Hill. 2008; $17^{\text {th }}$ ed., pp. 498.

15. Tourtellotte WG, Nagarajan R, Auyeung A, Mueller C, Milbrandt J: Infertility associated with incomplete spermatogenic arrest and oligozoospermia in Egr4-deficient mice. Development. 1999; 126: 5061-71.

16. Boisen KA, Main KM, Rajpert-De Meyts E, Skakkebaek NE: Are male reproductive disorders a common entity? The testicular dysgenesis syndrome. Ann N Y Acad Sci. 2001; 948: 90-9.

17. Lacerda HM, Akre O, Merletti F, Richiardi L: Time trends in the incidence of testicular cancer in childhood and young adulthood. Cancer Epidemiol Biomarkers Prev. 2009; 18: 2042-5.

18. Swan SH, Elkin EP, Fenster L: The question of declining sperm density revisited: an analysis of 101 studies published 1934-1996. Environ Health Perspect. 2000; 108: 961-6.
19. Paulozzi LJ: International trends in rates of hypospadias and cryptorchidism. Environ Health Perspect. 1999; 107: 297-302.

20. Vierula M, Niemi M, Keiski A, Saaranen M, Saarikoski S, Suominen J: High and unchanged sperm counts of Finnish men. Int J Androl. 1996; 19: 11-7.

21. Richiardi L, Bellocco R, Adami HO, Torrang A, Barlow L, Hakulinen T, et al.: Testicular cancer incidence in eight northern European countries: secular and recent trends. Cancer Epidemiol Biomarkers Prev. 2004; 13: 2157-66.

22. Boisen KA, Kaleva M, Main KM, Virtanen HE, Haavisto AM, Schmidt IM, et al.: Difference in prevalence of congenital cryptorchidism in infants between two Nordic countries. Lancet. 2004; 363: 1264-9.

23. Boisen KA, Chellakooty M, Schmidt IM, Kai CM, Damgaard IN, Suomi AM, et al.: Hypospadias in a cohort of 1072 Danish newborn boys: prevalence and relationship to placental weight, anthropometrical measurements at birth, and reproductive hormone levels at three months of age. J Clin Endocrinol Metab. 2005; 90: 4041-6.

24. Cortes D, Thorup JM, Visfeldt J: Cryptorchidism: aspects of fertility and neoplasms. A study including data of 1,335 consecutive boys who underwent testicular biopsy simultaneously with surgery for cryptorchidism. Horm Res. 2001; 55: 21-7.

25. Weidner IS, Møller H, Jensen TK, Skakkebaek NE: Risk factors for cryptorchidism and hypospadias. J Urol. 1999; 161: 1606-9.

26. Rajpert-De Meyts E: Developmental model for the pathogenesis of testicular carcinoma in situ: genetic and environmental aspects. Hum Reprod Update. 2006; 12: 303-23.

27. Bergström R, Adami HO, Möhner M, Zatonski W, Storm H, Ekbom A, et al.: Increase in testicular cancer incidence in six European countries: a birth cohort phenomenon. J Natl Cancer Inst. 1996; 88: 727-33.

28. Irvine S, Cawood E, Richardson D, MacDonald E, Aitken J: Evidence of deteriorating semen quality in the United Kingdom: birth cohort study in 577 men in Scotland over 11 years. BMJ. 1996; 312: 467-71.

29. Ge RS, Chen GR, Tanrikut C, Hardy MP: Phthalate ester toxicity in Leydig cells: developmental timing and dosage considerations. Reprod Toxicol. 2007; 23: 366-73.

30. Docimo SG, Silver RI, Cromie W: The undescended testicle: diagnosis and management. Am Fam Physician. 2000; 62: 2037-44, 2047-8.

31. Lu CC, Steinberger A: Effects of estrogen on human seminiferous tubules: light and electron microscopic analysis. Am J Anat. 1978; 153: 1-13. 
32. Fowler KA, Gill K, Kirma N, Dillehay DL, Tekmal RR: Overexpression of aromatase leads to development of testicular leydig cell tumors : an in vivo model for hormone-mediated TesticularCancer. Am J Pathol. 2000; 156: 347-53.

33. Skakkebaek NE, Rajpert-De Meyts E, Main KM: Testicular dysgenesis syndrome: an increasingly common developmental disorder with environmental aspects. Hum Reprod. 2001; 16: 972-8.

34. Weir HK, Marrett LD, Kreiger N, Darlington GA, Sugar L: Pre-natal and peri-natal exposures and risk of testicular germ-cell cancer. Int J Cancer. 2000; 87: 438-43.

35. Sharpe RM, Skakkebaek NE: Are oestrogens involved in falling sperm counts and disorders of the male reproductive tract? Lancet. 1993; 341: 1392-5.

36. Pierik FH, Burdorf A, Deddens JA, Juttmann RE, Weber RF: Maternal and paternal risk factors for cryptorchidism and hypospadias: a case-control study in newborn boys. Environ Health Perspect. 2004; 112 : 1570-6.

37. Andersen HR, Schmidt IM, Grandjean P, Jensen TK, Budtz-Jørgensen E, Kjaerstad MB, et al.: Impaired reproductive development in sons of women occupationally exposed to pesticides during pregnancy. Environ Health Perspect. 2008; 116: 566-72.

38. Suomi AM, Main KM, Kaleva M, Schmidt IM, Chellakooty M, Virtanen HE, et al.: Hormonal changes in 3-month-old cryptorchid boys. J Clin Endocrinol Metab. 2006; 91: 953-8.

39. Hsieh MH, Breyer BN, Eisenberg ML, Baskin LS: Associations among hypospadias, cryptorchidism, anogenital distance, and endocrine disruption. Curr Urol Rep. 2008; 9: 137-42.

40. Andersson AM, Jørgensen N, Frydelund-Larsen L, Rajpert-De Meyts E, Skakkebaek NE: Impaired Leydig cell function in infertile men: a study of 357 idiopathic infertile men and 318 proven fertile controls. J Clin Endocrinol Metab. 2004; 89: 3161-7.

41. Petersen PM, Giwercman A, Hansen SW, Berthelsen JG, Daugaard G, Rørth M, et al.: Impaired testicular function in patients with carcinoma-in-situ of the testis. J Clin Oncol. 1999; 17: 173-9.

42. Ferrara D, Hallmark N, Scott H, Brown R, McKinnell C, Mahood IK, et al.: Acute and long-term effects of in utero exposure of rats to di(n-butyl) phthalate on testicular germ cell development and proliferation. Endocrinology. 2006; 147: 5352-62.

43. Gray LE Jr, Wilson VS, Stoker T, Lambright C, Furr $\mathrm{J}$, Noriega $\mathrm{N}$, et al.: Adverse effects of environmental antiandrogens and androgens on reproductive devel- opment in mammals. Int J Androl. 2006; 29: 96-104; discussion 105-8.

44. Fisher JS, Macpherson S, Marchetti N, Sharpe RM: Human 'testicular dysgenesis syndrome': a possible model using in-utero exposure of the rat to dibutyl phthalate. Hum Reprod. 2003; 18: 1383-94.

45. Mahood IK, Scott HM, Brown R, Hallmark N, Walker M, Sharpe RM: In utero exposure to di(n-butyl) phthalate and testicular dysgenesis: comparison of fetal and adult end points and their dose sensitivity. Environ Health Perspect. 2007; 115 (Suppl 1): 55-61.

46. Møller H, Skakkebaek NE: Risk of testicular cancer in subfertile men: case-control study. BMJ. 1999; 318 : 559-62.

47. Petersen PM, Skakkebaek NE, Giwercman A: Gonadal function in men with testicular cancer: biological and clinical aspects. APMIS. 1998; 106: 24-34; discussion 34-6.

48. Olesen IA, Hoei-Hansen CE, Skakkebaek NE, Petersen JH, Rajpert-De Meyts E, Jørgensen N: Testicular carcinoma in situ in subfertile Danish men. Int J Androl. 2007; 30: 406-11; discussion 412.

49. Jacobsen R, Bostofte E, Engholm G, Hansen J, Olsen JH, Skakkebaek NE, et al.: Risk of testicular cancer in men with abnormal semen characteristics: cohort study. BMJ. 2000; 321: 789-92.

50. Raman JD, Nobert CF, Goldstein M: Increased incidence of testicular cancer in men presenting with infertility and abnormal semen analysis. J Urol. 2005; 174: 1819-22; discussion 1822.

Accepted:

January 29, 2010

\section{Correspondence address:}

Dr. Ashok Agarwal

Professor and Director

Center for Reproductive Medicine

Cleveland Clinic

9500 Euclid Avenue, Desk A19.1

Cleveland, Ohio, 44195, USA

Fax: 216-445-6049

E-mail: agarwaa@ccf.org 